\title{
Four new species of the genus Caccothryptus (Coleoptera, Limnichidae)
}

\author{
Hiroyuki YOSHITOMI \\ Ehime University Museum, Bunkyo 3, Matsuyama, 790-8577 Japan \\ E-mail: hymushi@agr.ehime-u.ac.jp \\ urn:1sid:zoobank.org:author:744FA6FC-2D84-41B7-8A6C-C1611CC68EE1
}

\begin{abstract}
Four new species of the genus Caccothryptus (Coleoptera: Limnichidae) are described: $C$. taiwanus from Taiwan; C. orion from Okinawa; C. tibetanus and C. chayuensis from Tibet. All the species belong to the testudo species group (sensu Hernando \& Ribera 2014). Additional specimen data and an updated species list are also given, and C. testudo Champion, 1923 is newly recorded from Thailand.
\end{abstract}

Key words. Limnichidae, taxonomy, distribution, new species.

Yoshitomi H. 2015. Four new species of the genus Caccothryptus (Coleoptera, Limnichidae). European Journal of Taxonomy 147: 1-17. http://dx.doi.org/10.5852/ejt.2015.147

\section{Introduction}

The family Limnichidae, minute marsh-loving beetles, is aquatic and semiaquatic, and represented by about 400 species belonging to 37 genera (Spangler et al. 2001; Hernando \& Ribera 2005b). The species diversity of this family is high in the Neotropical and the Oriental Regions (Hernando \& Ribera 2005b), but the Oriental fauna of this family has not been well studied.

The Oriental genus Caccothryptus Sharp, 1902 was recently revised (Hernando \& Ribera 2014), and 20 species subdivided into 5 species groups were recognized. In the present paper, I describe 4 additional new species from Okinawa, Taiwan and Tibet.

\section{Materials and methods}

General observations and dissections were made under a Leica MZ95 stereo microscope. Microstructures of dissected parts were studied in pure glycerine under an Olympus BH-2 compound microscope. After observation, the dissected parts were mounted on the same card as the specimen. Photographs were taken under a Leica MZ95, using a microscopy camera system (Nikon DS-Fi1-L2), and combined with automontage software Combine ZM (Alan Hadley, UK).

The specimens examined are preserved in the following museums:

EUMJ = Ehime University Museum, Matsuyama, Japan 
$\mathrm{NME}=$ Naturkundemuseum, Erfurt, Germany

$\mathrm{NMW}=$ Naturhistorisches Museum, Vienna

SEHU $=$ Hokkaido University Museum, Sapporo, Japan

TARI $=$ Taiwan Agricultural Research Institute, Taipei, Taiwan ROC

\section{Abbreviations}

Morphological abbreviations used for measurements are as follows:

$\mathrm{TL}=$ total length $(\mathrm{PL}+\mathrm{EL})$

$\mathrm{PL}=$ pronotal length in median line

$\mathrm{PW}=$ maximum width of pronotum

$\mathrm{EL}=$ elytral length in median line

$\mathrm{EW}=$ maximum width of elytra

The average is given in parentheses after the range.

\section{Results}

Class Hexapoda Blainville, 1816

Order Coleoptera Linnaeus, 1758

Suborder Polyphaga Emery, 1886

Series Elateriformia Crowson, 1960

Superfamily Byrrhoidea Latreille, 1804

Family Limnichidae Erichson, 1846

Subfamily Limnichinae Erichson, 1846

Genus Caccothryptus Sharp, 1902

Caccothryptus Sharp, 1902: 63. Hernando \& Ribera 2014: 283 [revision]. Type species: Caccothryptus compactus Sharp, 1902, by monotypy. urn:1sid:zoobank.org:act:CB7B4207-FD54-43A0-96177D15CAD62833

Macrobyrrhinus Pic, 1922: 4. Synonymised by Champion (1923: 222). Type species: Macrobyrrhinus rouyeri Pic, 1922, by monotypy. urn:lsid:zoobank.org:act:49DFB03F-B836-4673-8AF9-850B9E847115

\section{Remarks}

This genus is related to five genera in the Mandersia genus group (sensu Hernando \& Ribera 2005a): Resachus Delève, 1968 (revised by Hernando \& Ribera 2006), Simplocarina Pic, 1922, Pseudothryptus Hernando \& Ribera, 2005, Mandersia Sharp, 1902 and Euthryptus Sharp, 1902. It is also similar to Cyclolimnichus Delève, 1968 (revised by Hernando \& Ribera 2000) and Tricholimnichus Hernando \& Ribera, 2001 in their large and elongate body.

\section{Distribution}

SE Asia (Okinawa to India). 
Caccothryptus taiwanus sp. nov.

urn:Isid:zoobank.org:act:578B475A-54DA-4600-9FFF-F5E7045F814E

Figs $1 \mathrm{~A}, 2 \mathrm{~A}-\mathrm{E}, 4 \mathrm{~A}-\mathrm{B}$

\section{Diagnosis}

Smaller species in the genus; $\mathrm{TL}=3.73-4.05 \mathrm{~mm}$ in male, $3.75-4.20 \mathrm{~mm}$ in female; parameres weakly pointed in lateral view; apical emargination of parameres deeply U-shaped, with small projections in inner margins.

\section{Etymology}

The species is named after the type locality.

\section{Material examined}

\section{Holotype}

TAIWAN: ${ }^{\top}$ (TARI), “Taiwan: Ilan Fushan ---- [Chinese characters] 13. IV. 2011, leg. C. F. Lee”.

\section{Paratypes}

TAIWAN: 2 of, 7 우, 13 ex. (TARI, EUMJ, NMW), same data as for the holotype; 1 ex. (TARI),

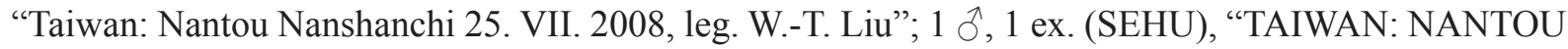
Nanshanchi 900 28. III. 1982 Col. T. FUJISAWA"; 1 § (EUMJ), "[TAIWAN] Wulu Alt. ca. $700 \mathrm{~m}$ Haiduan Township Taitung Country ---- [Chinese characters] 17-18. VI. 2011 J. Yamasako leg."; 1 q (EUMJ), "(TAIWAN) Hsinhsien Wulai Taipei Hsien 2. VII, 1970 Y. Hori leg.”.

\section{Description}

\section{Male}

Body (Fig. 1A) oblong, convex dorsally and ventrally, shiny, densely covered with short and long silver setae. Coloration of body black; legs and antennae paler.

Head densely punctate, slightly convex dorsally. Antennae relatively long, reaching about proximal 1/4 of elytra. Pronotum punctate as in head; PW/PL 1.90-2.11 (2.03). Scutellar shield equilateral triangular, finely punctate, lateral margins straight. Elytra oblong, widest at middle, lateral margins gently arcuate, coarsely and irregularly punctate; space between punctures same as their diameter; adpressed long silver setae forming obvious zigzag, irregular markings; apex obtuse; humeral parts weakly projecting dorsally; EL / EW 1.17-1.37 (1.31), EL / PL 3.00-3.50 (3.26), EW / PW 1.17-1.37 (1.23), TL / EW $1.53-1.78$ (1.72). Each claw on foreleg of same size and shape.

Sternite VIII (Fig. 2D) U-shaped, bearing short setae in apical parts. Sternite IX (Fig. 2E) elongate, with long and stout lateral projections. Aedeagus (Fig. 2A-C) stout, straightly curved ventrally in apical part, punctate in apical part of median lobe and parameres; median lobe wide in lateral view, rather pointed at apex; apical emargination of parameres deeply U-shaped, with small projections in inner margins; apices of parameres rounded in ventral view, weakly pointed in lateral view.

\section{Female}

Sexual dimorphism indistinct; PW / PL 1.65-2.26 (2.02), EL / EW 1.23-1.34 (1.30), EL / PL 2.65-3.56 (3.25), EW / PW 1.20-1.28 (1.23), TL / EW 1.60-1.79 (1.71). Ovipositor (Fig. 4B) well sclerotized; coxite closely punctuate, pointed at apices, about 1.18 times as long as spiculum ventrale (Fig. 4A); approximate ratio of coxite and baculus $(\mathrm{n}=1) 1.0: 3.43$. 


\section{Measurements}

Males (n= 5): TL 3.73-4.05 (3.90) mm, PW 1.80-1.90 (1.86) mm, PL 0.88-1.00 (0.92) mm, EL 2.853.15 (2.98) mm, EW 2.10-2.50 (2.28) $\mathrm{mm}$.

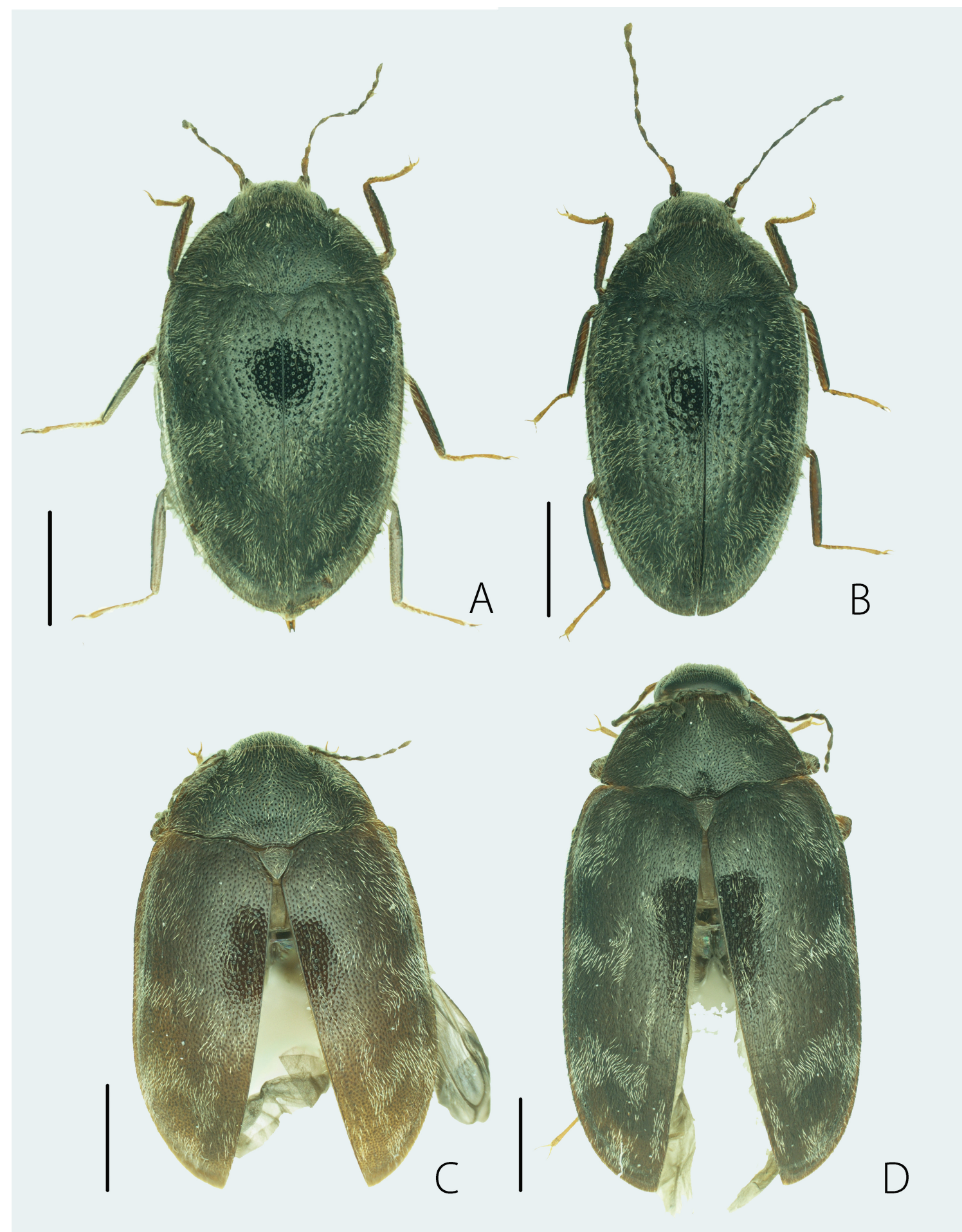

Fig. 1. Habitus of Caccothryptus spp., holotypes, $\hat{\partial}$. A. Caccothryptus taiwanus sp. nov. B. C. orion sp. nov. C. C. tibetanus sp. nov. D. C. chayuensis sp. nov. Scales $=1.0 \mathrm{~mm}$. 
Females $(\mathrm{n}=8)$ : TL 3.75-4.20 (4.02) mm, PW 1.80-2.03 (1.90) mm, PL 0.85-1.15 (0.95) mm, EL 2.90-3.20 (3.07) mm, EW 2.20-2.50(2.35) mm.

\section{Distribution}

Only known from the type locality, Taiwan.
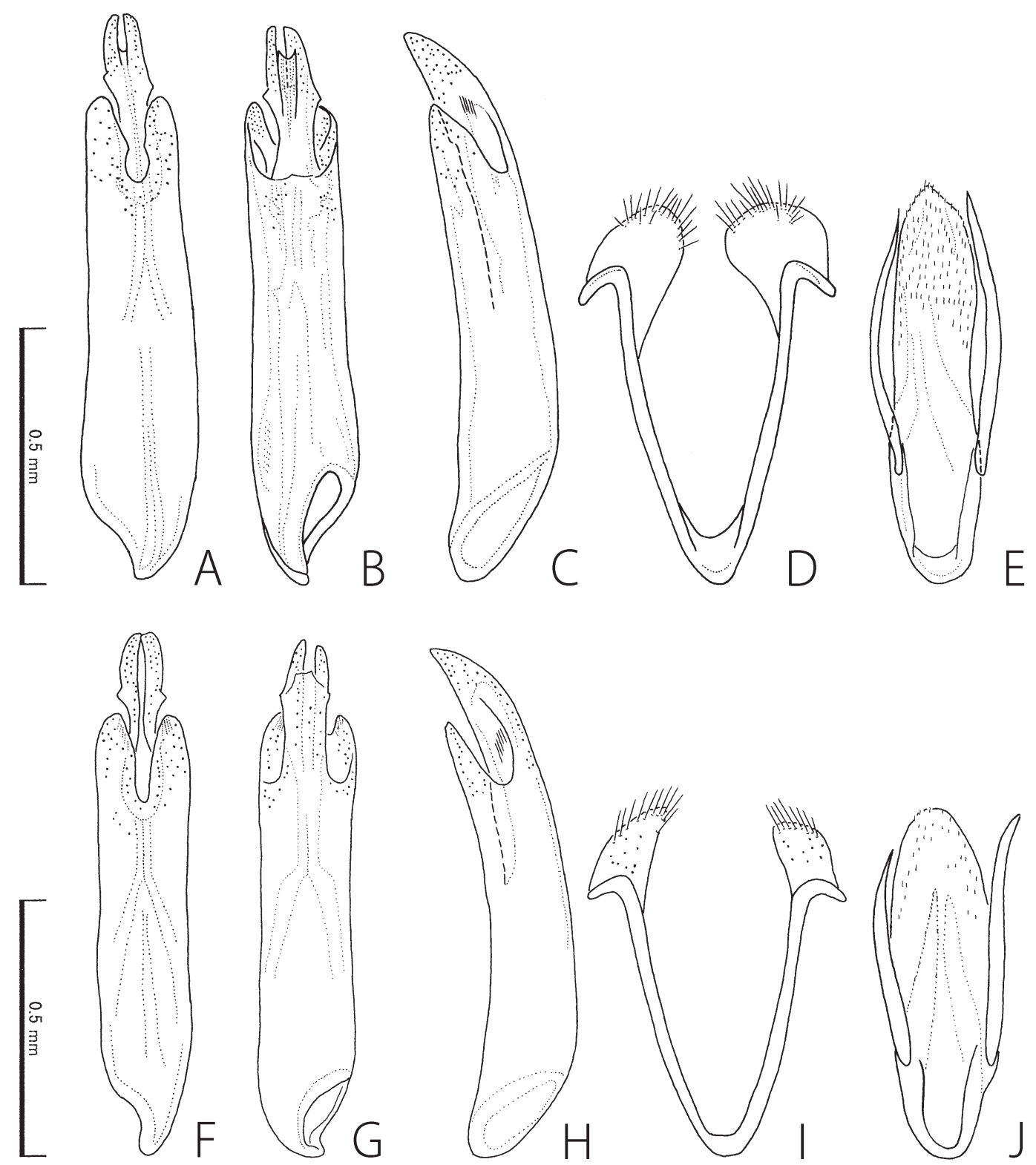

Fig. 2. Male genitalia of Caccothryptus spp. A-E. Caccothryptus taiwanus sp. nov. F-J. C. orion sp. nov. - A-C, F-H. Aedeagus in ventral (A, F), dorsal (B, G) and lateral (C, H) views. D, I. Sternite VIII. E, J. Sternite IX. 


\section{Remarks}

This species belongs to the testudo species group (sensu Hernando \& Ribera 2014). It resembles $C$. sinensis Hernando \& Ribera, 2014, known from Fujian, China, in the shape of the parameres, which are broadly and deeply emarginated, and differs from it in the following male genital characteristics: apex of the median lobe rather rounded (rather pointed in $C$. sinensis); median lobe straightly projecting posteriorly (curved postero-ventrally in C. sinensis).

Caccothryptus orion sp. nov. urn:1sid:zoobank.org:act:42B7D5F0-7DC5-4BD5-8CCF-5A08F9222A54

[Japanese name: Okinawa-oo-chibi-doromushi]

Figs $1 \mathrm{~B}, 2 \mathrm{~F}-\mathrm{J}, 4 \mathrm{C}-\mathrm{D}, 5 \mathrm{~A}-\mathrm{B}$

\section{Diagnosis}

Smaller species in the genus; $\mathrm{TL}=3.68-3.73 \mathrm{~mm}$ in male, $3.82-4.00 \mathrm{~mm}$ in female; parameres rather pointed in lateral view, with furrows in ventral part; apical emargination of parameres deeply U-shaped.

\section{Etymology}

The species is named after "Orion Beer" (copyright: Orion Breweries, Ltd), which is a local, but wellknown and favored beer in Okinawa. The type locality of this species is situated near the Nago Factory of Orion Beer.

\section{Material examined}

Holotype

JAPAN: 1 ภे (EUMJ), “Genkagawa, Nago-shi, Okinawa Japan N26.616704, E128.062778, 10-IV-2015, Masato MORI leg.".

\section{Paratypes}

JAPAN: 1 (EUMJ), same data as for the holotype; 1 , 1 ex. (EUMJ), same data, but collector K. Kitayama; 1 ㅇ (EUMJ), “Zatsun, Kunigami, Okinawa, Ryukyus 21-VI-2006 K. Takahashi leg."; 2 우 (EUMJ), "Genka-ohkawa Okinawa 22-VII-1996 C. F. Lee leg.”.

\section{Description}

Male

Very similar to $C$. taiwanus sp. nov. in external features; body a little smaller and slender; elytral punctures coarser; PW / PL 1.88-1.94 (1.91), EL / EW 1.39-1.42 (1.40), EL / PL 3.14-3.33 (3.24), EW / PW 1.16-1.25 (1.21), TL / EW 1.83-1.84 (1.84).

Sternite VIII (Fig. 2I) U-shaped, bearing short setae in apical parts. Sternite IX (Fig. 2J) rather wide, with long and stout lateral projections. Aedeagus (Fig. $2 \mathrm{~F}-\mathrm{H}$ ) stout, curved ventrally in apical part, punctate in apical part of median lobe and parameres; median lobe wide in lateral view, pointed at apex; apical emargination of parameres deeply U-shaped; apices of parameres rounded in ventral view; pointed in lateral view.

\section{Female}

Sexual dimorphism indistinct; PW / PL 1.85-1.94 (1.91), EL / EW 1.36-1.45 (1.42), EL / PL 3.15-3.44 (3.33), EW / PW 1.18-1.29 (1.23), TL / EW 1.77-1.91 (1.84). Ovipositor (Fig. 4D) well sclerotized; coxite closely punctuate, pointed at apices, about 1.11 times as long as spiculum ventrale (Fig. 4C); approximate ratio of coxite and baculus $(\mathrm{n}=1)$ as $1.0: 3.45$. 


\section{Measurements}

Males $(\mathrm{n}=2)$ : TL 3.68-3.73 (3.71) mm, PW 1.60-1.75 (1.68) mm, PL 0.85-0.90 (0.88) mm, EL 2.83 $\mathrm{mm}$, EW 2.00-2.03 (2.02) $\mathrm{mm}$.

Females $(\mathrm{n}=4)$ : TL 3.82-4.00 (3.92) mm, PW 1.70-1.75 (1.73) mm, PL 0.90-0.92 (0.91) mm, EL 2.90-3.10 (3.01) mm, EW 2.00-2.20 (2.13) mm.

\section{Distribution}

Only known from the type locality, Okinawa-jima, the Ryukyus, Japan.

\section{Remarks}

This species belongs to the testudo species group (sensu Hernando \& Ribera 2014). It is similar to $C$. taiwanus sp. nov. in the shape of the male genitalia, but differs from it in the following characteristics: parameres rather pointed in lateral view, with furrows in the ventral part; apex of the median lobe rather pointed in lateral view.

\section{Biological notes}

The type locality (Fig. 5A-B) is a small river and the specimens were collected from under the surface of water-logged wood (personal communication from Mr. Masato Mori).

Caccothryptus tibetanus sp. nov. urn:1sid:zoobank.org:act:3CE4012A-96D7-4568-9DB7-F2175F0B1278

Figs $1 \mathrm{C}, 3 \mathrm{~A}-\mathrm{D}, 4 \mathrm{E}-\mathrm{F}$

\section{Diagnosis}

Medium size in the genus; $\mathrm{TL}=4.65 \mathrm{~mm}$ in male, $4.40-4.70 \mathrm{~mm}$ in female; apical emargination of parameres deeply U-shaped.

\section{Etymology}

The species is named after the type locality.

\section{Material examined}

\section{Holotype}

TIBET: ${ }^{\lambda}$ (EUMJ), “Cha Yu, 1824 m S.E. Tibet 9-IX-1996 C. I. Li leg.”.

\section{Paratypes}

TIBET: 3 q $q$ (EUMJ, NMW), same data as for the holotype.

\section{Description}

\section{Male}

Body oblong, convex strongly in dorsal and slightly in ventral parts, shiny, densely covered with short silver setae. Coloration of body dark brown, but femora and tarsi paler.

Head slightly convex dorsally, densely covered with fine punctures. Antennae relatively long, reaching about proximal 1/5 of elytra. Pronotum punctate as in head; PW / PL 2.10. Scutellar shield equilateral triangular, finely punctate, lateral margins straight. Elytra oblong, subparallel-sided near base to apical $1 / 4$, gently tapering in apical $1 / 4$, densely and regularly punctate; space between punctures smaller than their diameter; adpressed silver setae forming zigzag markings; apex relatively pointed; humeral parts 
distinctly projecting dorsally; EL / EW 1.40, EL / PL 3.65, EW / PW 1.24, TL / EW 1.79. Each claw on forelegs of same size and shape.
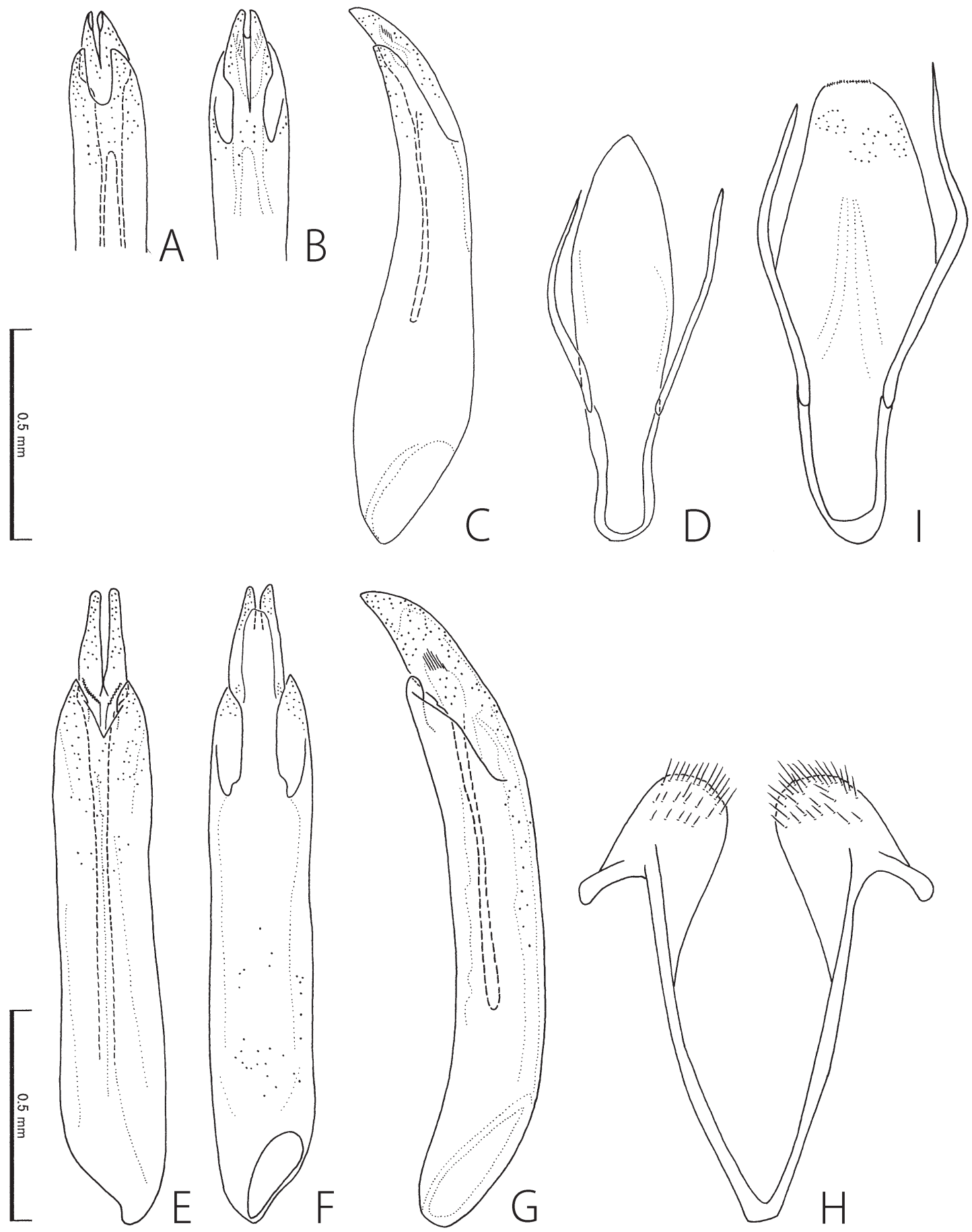

Fig. 3. Male genitalia of Caccothryptus spp. A-D. Caccothryptus tibetanus sp. nov. E-I. C. chayuensis sp. nov. - A-C, E-G. Aedeagus in ventral (A, E), dorsal (B, F) and lateral (C, G) views. H. Sternite VIII. D, I. Sternite IX. 
Sternite IX (Fig. 3D) elongate, with long and slender lateral projections. Aedeagus (Fig. 3A-C; basal part somewhat damaged) stout, curved ventrally in apical part, finely punctate in apical part of median lobe and parameres; median lobe slender in lateral view, pointed at apex; apical emargination of parameres deeply U-shaped; apices of parameres pointed in ventral and lateral views.

\section{Female}

Sexual dimorphism indistinct; PW / PL 1.98-2.08 (2.02), EL / EW 1.32-1.42 (1.37), EL / PL 3.40-3.76 (3.62), EW / PW 1.24-1.35 (1.31), TL / EW 1.68-1.80 (1.76). Ovipositor (Fig. 4F) well sclerotized; coxite closely punctuate, pointed at apices, about 1.03 times as long as spiculum ventrally (Fig. 4E); approximate ratio of coxite and baculus $(\mathrm{n}=1) 1.0: 3.41$.

\section{Measurements}

Male (n= 1): TL 4.65 mm, PW 2.10 mm, PL 1.00 mm, EL 3.65 mm, EW 2.60 mm.

Females $(\mathrm{n}=3)$ : TL 4.40-4.70 (4.59) mm, PW 1.95-2.08 (2.00) mm, PL 0.98-1.00 (0.99) mm, EL 3.40-3.70 (3.59) mm, EW 2.45-2.80 (2.62) mm.

\section{Distribution}

Only known from the type locality, Tibet.

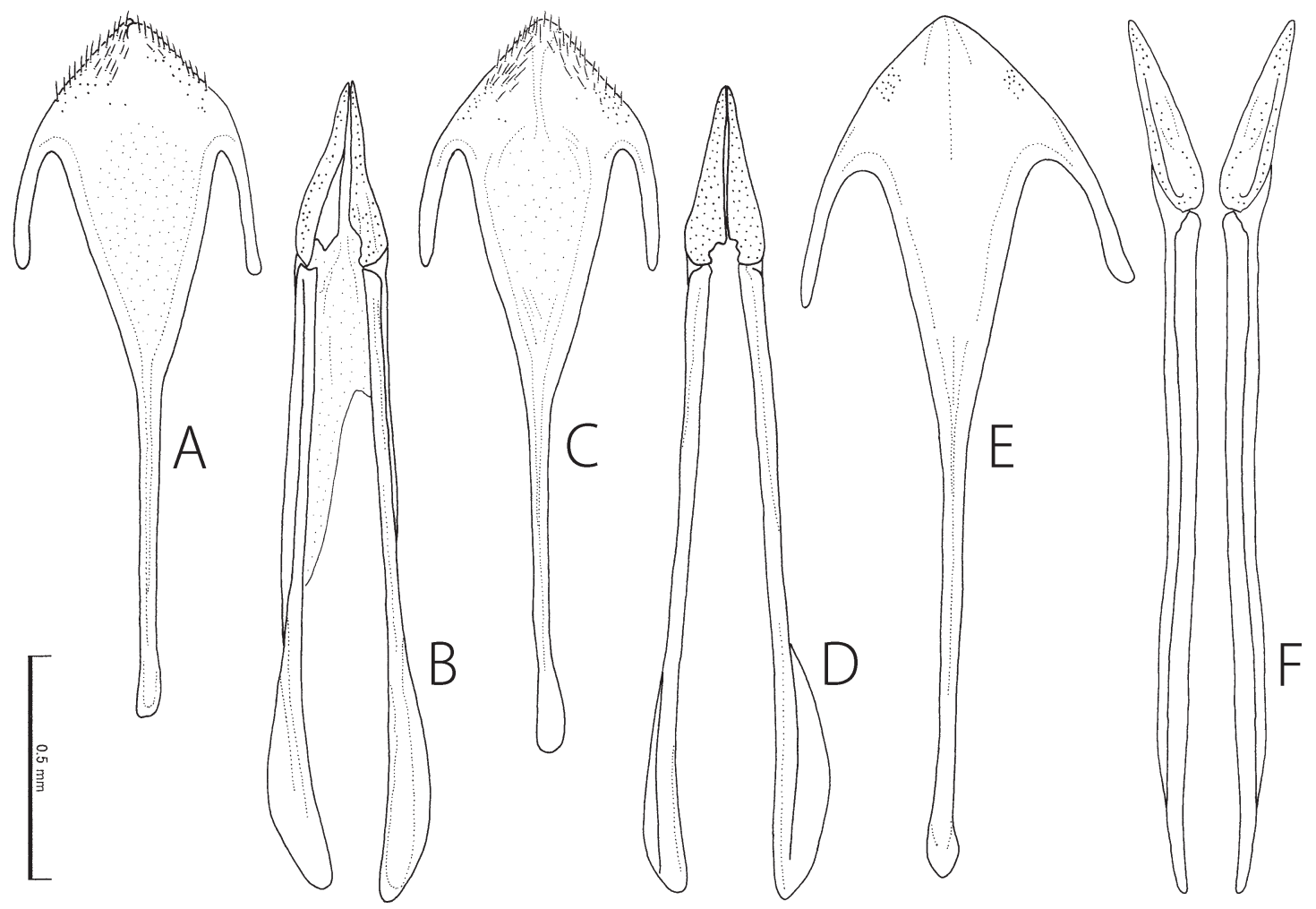

Fig. 4. Female genitalia of Caccothryptus spp. A-B. Caccothryptus taiwanus sp. nov. C-D. C. orion sp. nov. E-F. C. tibetanus sp. nov. - A, C, E. Spiculum ventrale. B, D, F. Ovipositor. 


\section{Remarks}

This species belongs to the testudo species group (sensu Hernando \& Ribera 2014). It is related to C. sinensis, but differs from it in having the median lobe straightly projecting posteriorly, and the emargination of parameres somewhat wider.

Caccothryptus chayuensis sp. nov. urn:1sid:zoobank.org:act:43136CDF-3F5C-4AD4-A7DC-3F57299013EC

Figs 1D, 3E-I

\section{Diagnosis}

Larger species in the genus; $\mathrm{TL}=5.90 \mathrm{~mm}$ in male; median lobe minutely serrate in ventral part; apical emargination of parameres V-shaped.

\section{Etymology}

The species is named after the type locality.

\section{Material examined}

Holotype

TIBET: ${ }^{\lambda}$ (EUMJ), “Cha Yu, 1824m S.E. Tibet 9-IX-1996 C. I. Li leg.”.

\section{Description}

Male

Body oblong, convex strongly in dorsal and slightly in ventral parts, shiny, densely covered with short silver setae. Coloration of body dark brown, but femora and tarsi paler.

Head convex dorsally, densely covered with fine punctures. Antennae relatively long, reaching about proximal $1 / 5$ of elytra. Pronotum punctate as in head; PW / PL 2.04. Scutellar shield equilateral triangular, finely punctate, lateral margins straight. Elytra oblong, subparallel-sided near base to apical $1 / 4$, gently tapering in apical 1/4, relatively pointed at apex, sparsely and irregularly punctate; space between punctures larger than their diameter; adpressed silver setae forming zigzag markings; humeral parts distinctly convex dorsally; EL / EW 1.50, EL / PL 4.17, EW / PW 1.36, TL / EW 1.86. Each claw on foreleg of same size and shape.

Sternite VIII (Fig. 3H) U-shaped, bearing short setae in apical parts. Sternite IX (Fig. 3I) elongate, with long and slender lateral projections. Aedeagus (Fig. 3E-G) slender, slightly curved ventrally, finely punctate in apical part of median lobe and parameres; median lobe wide in lateral view, rather pointed at apex, minutely serrate in ventral part; apical emargination of parameres V-shaped; apices of parameres pointed in ventral view, forming thumb-like projection in lateral view.

\section{Female}

Unknown.

\section{Measurements}

Male (n=1): TL 5.9 mm, PW 2.35 mm, PL 1.15 mm, EL 4.80 mm, EW 3.20 mm.

\section{Distribution}

Only known from the type locality, Tibet. 


\section{Remarks}

This species belongs to the testudo species group (sensu Hernando \& Ribera 2014). It is related to $C$. fujianensis Hernando \& Ribera, 2014 known from China (Fujian), C. malickyi Hernando \& Ribera, 2014 from Vietnam and C. jendeki Hernando \& Ribera, 2014 from India, but differs from them in the following characteristics: median lobe with minute serrae in the ventral part, pointed at apex; emargination of parameres rather shallow.

\section{Specimens examined for comparison}

The compactus species group

Caccothryptus maculosus (Pic, 1923)

\section{Material examined}

LAOS: 1 đે, 2 ex. (EUMJ), “20km NE of Vang Vieng, Viangchan Prov., C. LAOS 23.III.2005 M. Sato leg."

\section{Measurements $(\mathrm{n}=3)$}

TL 4.65-5.30 (4.95) mm, PW 2.00-2.35 (2.20) mm, PL 1.05-1.20 (1.13) mm, EL 3.60-4.10 (3.82) mm, EW 2.55-2.90 (2.70) mm, PW / PL 1.90-1.96 (1.94), EL / EW 1.41-1.42 (1.41), EL / PL 3.26-3.43 (3.37), EW / PW 1.18-1.28 (1.23), TL / EW 1.82-1.85 (1.83).

\section{Caccothryptus sulawesianus Hernando \& Ribera, 2014}

\section{Material examined}

INDONESIA: 1 क (EUMJ), “C. Sulawesi, Palopo, Kilo Lima Belas, Battang, Wara Barat alt. 300 m, 2-II-2013 Kiyoshi Ando leg. S0257' E12007”’.

\section{Measurements}

Female (n = 1): TL 4.37 mm, PW 1.95 mm, PL 1.00 mm, EL 3.37 mm, EW 2.45 mm, PW / PL 1.95, EL / EW 1.38, EL / PL 3.37, EW / PW 1.26, TL / EW 1.78.

\section{Remarks}

Judging from the collecting locality, I determined the species.

The testudo species group

Caccothryptus auratus Hernando \& Ribera, 2014

\section{Material examined}

THAILAND: 1 ภ, 5 ex. (EUMJ), "[North THAI] Maeo Khun Klang 1350 m, Doi Inthanon 20. X. 1983 M. Sakai”; 1 ex. (EUMJ), "at light", “(THAILAND) Maeo Khun Klang, 1300 m Doi Inthanon 17. X. 1983 M. Tomokuni".

\section{Measurements}

$(\mathrm{n}=7)$ : TL $3.55-3.75(3.67) \mathrm{mm}$, PW 1.70-1.82 (1.78) mm, PL 0.75-0.85 (0.81) mm, EL 2.75-2.95 (2.87) mm, EW 2.15-2.30 (2.21) mm, PW / PL 2.12-2.31 (2.21), EL / EW 1.28-1.32 (1.30), EL / PL 3.39-3.77, (3.56), EW / PW 1.22-1.26 (1.24), TL / EW 1.63-1.70 (1.66). 


\section{Caccothryptus malickyi Hernando \& Ribera, 2014}

\section{Material examined}

VIETNAM: 1 đ, 1 \& (EUMJ; Fig. 5C-D), “[VN5] Ban Khoang, Lao Cai Prov., Vietnam, N22 23.329 E103 47.093, ca 1675 m, 22. VI. 2012, H. Yoshitomi leg."; 5 ex. (EUMJ), "Sapa (alt. 1500 m) Lao Cai Prov. VIETNAM 12. X. 1994 M. Satô leg."; 1 Oૈ, 1 q, 1 ex. (EUMJ), "Pass north of Mt. Phang Si Pang (alt. 1700-1850m) N. VIETNAM 9. X. 1994 M. Satô leg.”.

\section{Measurements}

( $\mathrm{n}=10)$ : TL 5.05-6.20 (5.76) mm, PW 2.20-2.68 (2.45) mm, PL 1.05-1.35 (1.21) mm, EL 3.90-4.85 (4.55) mm, EW 2.60-3.20 (3.00) mm, PW / PL 1.93-2.17 (2.02), EL / EW 1.44-1.57 (1.52), EL / PL 3.39-3.96 (3.75), EW / PW 1.17-1.28 (1.23), TL / EW 1.83-1.98 (1.92).
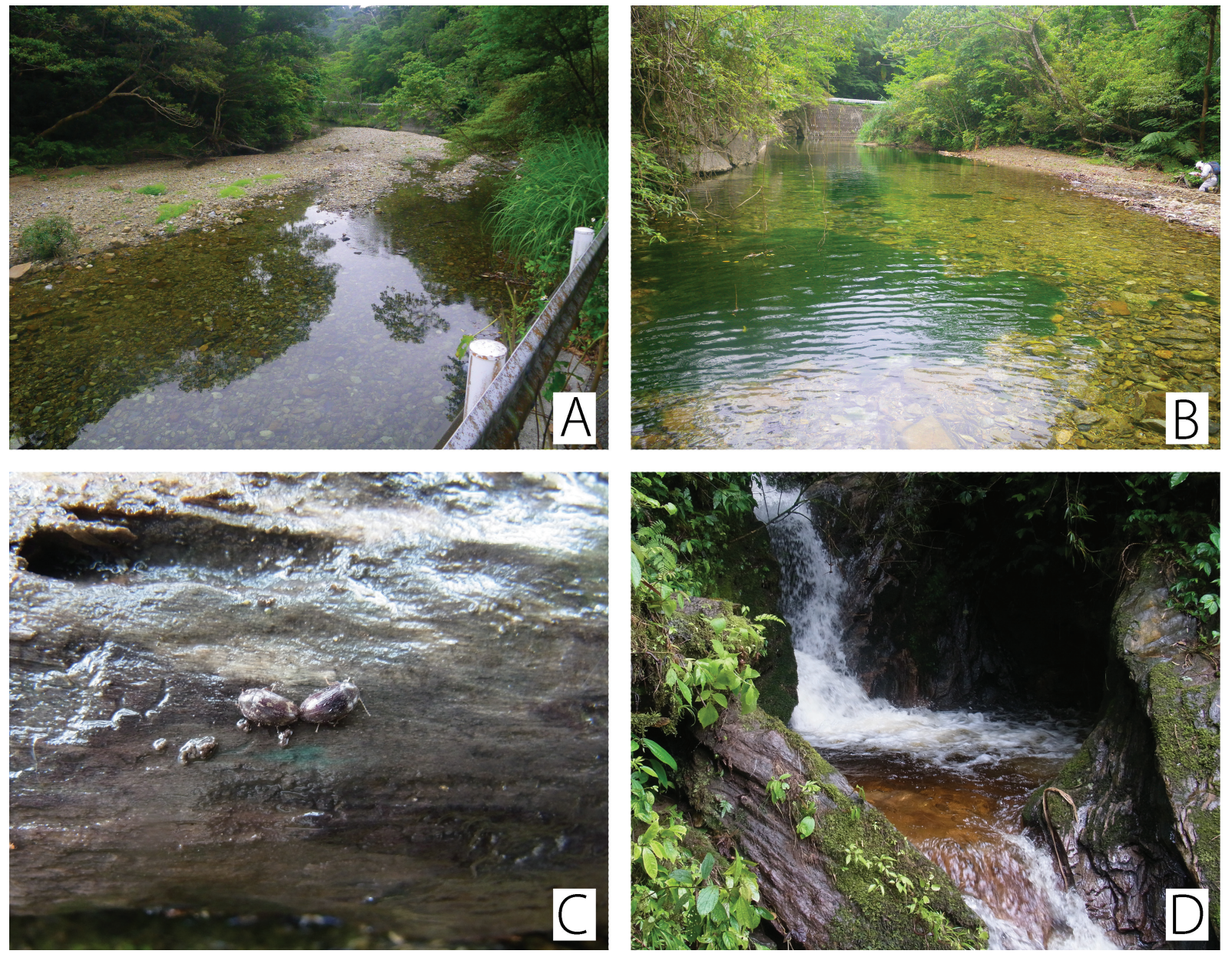

Fig. 5. Habitat of Caccothryptus spp. A-B. Genkagawa, Nago-shi, Okinawa, type locality of C. orion sp. nov. Photographs by Mr Mori. C-D. Ban Khoang, Vietnam, copulating individuals of C. malickyi Hernando \& Ribera, 2014 under surface of water-logged wood (C) and their environment (D). Photographs by author. 


\section{Caccothryptus ripicola Champion, 1923}

\section{Material examined}

NEPAL: 1 ठ (NME), "NEPAL, Annapurna Region, Umg. Bhulbhule 870m NN, 19. IX. 1992 leg. A. Wegel”; 1 ex. (NME), "NEPAL, Pokhara Südufer des Phewa Sees, Bachlauf, Gesiebe, 800-900 m 8. 5. 2001, leg. G. Hirthe".

\section{Measurements}

Sex unknown (n = 2): TL 3.92-4.00 (3.96) mm, PW 1.80-1.85 (1.83) mm, PL $0.90 \mathrm{~mm}$, EL 3.02-3.10 (3.06) mm, EW 2.30-2.35 (2.33) mm, PW / PL 2.00-2.06 (2.03), EL / EW 1.31-1.32 (1.32), EL / PL 3.36-3.44 (3.40), EW / PW 1.24-1.31 (1.27), TL / EW 1.70.

\section{Caccothryptus testudo Champion, 1923}

\section{Material examined}

NEPAL: 1 ex. (SEHU), "NEPAL: Bagmati TAKAGI-S.", "Syabru Ghora Tobela Sept. 22 '75”; 17 ex. (EUMJ), "Nangarpa 2000 m Sindhu, Nepal 11-XI-1979 M. Sato leg."; 1 ex. (EUMJ), "Soluke 2300 m Solukhumbu, Nepal 6-X-1979 M. Sato leg."; 11 ex. (EUMJ), "Khrte Danda 2700 m Solukhumbu, Nepal 7-X-1979 M. Sato leg."; 14 ex. (EUMJ), "Shivinokhola, 1920 m Sindhu, Nepal 14-XI-1979 M. Sato leg."; 3 ex. (EUMJ), "(EAST NEPAL) Kabre 1700-1760 m Dolakha Dist., 17. Oct., 1979 M. Tomokuni leg."; 171 ex. (NME, EUMJ, NMW), "NEPAL, Manaslu Mts. Bara Pokhari Lekh Chhandi Khola Valley 2000-220 [!] m NN, 11./12. IV. 2003, leg. J. Schmidt"; 5 ex. (NME), "NEPAL, Manaslu

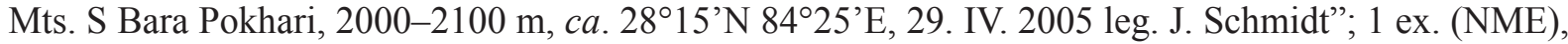
“NEPAL Manaslu Mts. SE-slope W Gupchi Danda 2200-2300 m, 2808’37N 8444'42E, 18 5. 2006, leg. J. Schmidt"; 2 ex. (NME), "NEPAL, Manaslu Mts 22. IV. 2003, Dudh Pokhari Leku, below Helam Pokhari, 2000 m NN, leg. J. Schmidt"; 2 ex. (NME), "NEPAL, Manaslu Mts. E slope of Ngadi Khola

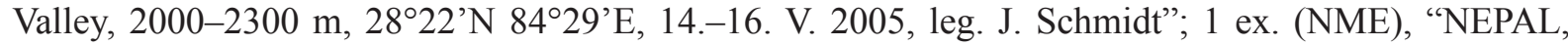
Manaslu Mts. 1600-1800 m NN, Bara Pokhari Lekh, above Bhachok Gaon vill., 29. IV. 2005, leg. Schmidt 2814'28'N, 8424'32'E”; 1 ex. (NME), "NEPAL, Manaslu Mts Du'dh Pokhari Lekh 21002500 m, NN upper Phulinggiri Mardi, 19-21. IV. 2003, leg. J. Schmidt"; 12 ex. (NME), "NEPAL, SW Dhaulagari Himal Dhara Khola Vali., 28³0’36”N, 83¹8’16”E, 1900 m, 21./22. V. 2012 leg. Schmidt"; 1 ex. (NME), "NEPAL, Phaulagiri SE slope, upp. Ru-hagat Khola, betw Chima Khola \& Dwari 10. V. 2002, 1750 m NN leg. J. Schmidt"; 3 ex. (NME), "NEPAL S Dhaulagiri Mts. Dhara Khola, 2100-2200 m, 10. V. 2012, leg. J. Schmidt, $28^{\circ} 31^{\prime} N$ 8317’E“; 6 ex. (NME), “C-NEPAL, Bagmati Kathmandu, Chaubas 2200 m, 19.-20. VI. 1989, leg. C. Holzschuh"; 4 ex. (NME), "NEPAL, Kathmandu Region oberhalb Godawari Kunako Khola gesiebt, 1750 m, 06. 05. 2001, leg. Hirthe"; 1 ex. (NME), "NEPAL, Kathmandu, N Shivapuri Lekh, 1700-1800 m, NN upper Bagmati riv. Valley, 24. V. 2005, leg. Schmidt";

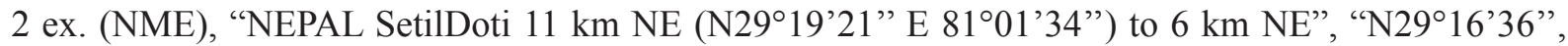
E8059'54" Dipayal, 2000-1000 m, 4. VII. 2009, leg. A. Weigel \#46"; 2 ex. (NME), "NEP: Mahakali/ Darchula $1 \mathrm{~km}$ NE Batar, Chamliya Khola, 2100 m, 29॰51'29”'N 8054’34”E, 11. VI. 2005 leg. A. Weigel LF"; 2 ex. (NME), "E-Nepal, Dhankuta Arun Valley, 23. V. 1980, Arunthan Chichila", "13001900 m leg. C. Holzschuh"; 5 ex. (NME), "NEPAL, Rolwaling Dolakha, Simigau to Dugong Kharka 2100 m NN, 16. V. 2000 leg. J. Schmidt"; 4 ex. (NME), "Madi Khola below Sikl 1500 m, 4. 8.", "NEPAL HIMALAYA Manaslu-Mts, Lg. Schmidt 1995"; 3 ex. (NME), "Meme Pokhari Lekh Upp. Taksar vill., 2100 m, 31. 8.", "Nepal HIMALAYA Annapurna-Mts. Lg. Schmidt 1995"; 1 ex. (NME), "Umgeb. Jiri 1707 m, Shivalaya 1. 5. Kimti Khola", "NEPAL-HIMALAYA s Khumbu-Himal lg. Kleeberg 1993"; 1 ex. (NME), "NEPAL, Longtang Syabru, Bamboo Lodge 2160-1900 m, 2809'N 85²4'E, 14. IX. 1997, leg. Fabrizi \& Ahrens"; 1 ex. (NME), "NEPAL, Prov. Mahakali Shinae bis Lager am Chamliya Khola bei Batar, 2000 m, 11. VI. 2005, leg.: J. Wei Pert"; 1 ex. (NME), "NEPAL, Baglung Lekh, ca. 
30 km W Baglung, N Tara Khola, 25-2700 m NN”, “18. V. 2004, leg. J. Schmidt, 28²2’N 83²0’E”; 1 ex. (NME), "Kali Gandaki Tal bei Tatopani, ca. 1000 m, 13. 6.", "NEPAL-HIMALAYA Annapurna Mts, 1993, lg. Schmidt"; 1 ex. (NME), "NEPAL, Prov. Gandaki Sikles range, water-power station, 1450 m NN, 10. 05. 1996, leg. J. Schmidt"; 1 ex. (NME), "NEPAL Annapurna Region Tikhendhunga, 1500 m, $28^{\circ} 20^{\prime} 93^{\prime}$ N, 8344'53”'E, 20. 04. 2000, leg. A. Skale, Fluß”; 1 ex. (NME), “NEPAL Annapurna Telbrung Danda, 2000 m, 15. 6. 97, leg. Schmidt"; 1 ex. (NME), "Annapurna Himal, Sikles range betw. 1400-2100 m, V. 1996, leg. Schmidt"; 1 ex. (NME), "NEPAL, Annapurna Region West Mardi Himal. Modi Khola Tal oberh. Himalpani, 1420-1480 m, 16. 05. 2001, leg. Hirthe"; 1 ex. (NME), "NEPAL, Annapurna Mts. Baglung Lekh, ca. 18 km W Baglung, upp. Okhle village, 2300 m, NN 13. V. 2004, leg. J. Schmidt"; 2 ex. (NME), "NEPAL, Annapurna South Himal, betw Khopra and Nanche, 2250-2350 m, NN 25./26. V. 2001 leg. J. Schmidt".

THAILAND: "1 $q$ (EUMJ), "at light", Doi Inthanon 1,670 m alt. 21. X. 1983 M. Owada"; 1 ภ, 1 q (EUMJ), “[North THAI] Doi Inthanon, 1640 m 18. X. 1983 M. Sakai leg.”; 1 \& (EUMJ), “(THAILAND) Maeo Khun Klang, 1300 m Doi Inthanon 16. X. 1983 M. Tomokuni”; 1 \& (EUMJ), "[North THAI] Maeo Khun Klang 1350 m, Doi Inthanon 19. X. 1983 M. Sakai"; 1 ex. (EUMJ), as preceding, but 20. X. 1983.

\section{Measurements}

Males ( $\mathrm{n}=19)$ : TL 4.70-5.42 (5.12) mm, PW 2.05-2.55 (2.25) mm, PL 1.00-1.20 (1.09) mm, EL 3.70-4.28 (4.02) mm, EW 2.30-2.85 (2.61) mm, PW / PL 1.86-2.32 (2.06), EL / EW 1.42-1.62 (1.54), EL / PL 3.46-3.89 (3.69), EW / PW 1.11-1.23 (1.16), TL / EW 1.82-2.06 (1.96).

Females (n = 17): TL 4.70-5.30 (5.06) mm, PW 2.00-2.35 (2.22) mm, PL 1.00-1.15 (1.07) mm, EL 3.70-4.20 (3.99) mm, EW 2.40-2.75 (2.63) mm, PW / PL 1.87-2.20 (2.08), EL / EW 1.41-1.62 (1.52), EL / PL 3.30-4.00 (3.73), EW / PW 1.13-1.26 (1.18), TL / EW 1.81-2.04 (1.93).

\section{Remarks}

This species is recorded from India and Nepal, and this is the first record from Thailand.

\section{Caccothryptus sp.}

\section{Material examined}

INDIA: 1 q (EUMJ), “ASSAM 5mi. W Digboi 110 m X-15-61", "E. S. Ross D. Cavagnaro Collector".

\section{Discussion}

Including the species described in this paper, it is now established that 24 species of the genus Caccothryptus are distributed in Japan (Okinawa), Taiwan, China, Tibet, Vietnam, Thailand, Nepal, Cambodia, India, the Philippines, Malaysia and Indonesia (see also appendix). The distribution of Caccothryptus tibetanus sp. nov. and C. chayuensis sp. nov. in Tibet is the northernmost record of the genus.

\section{Acknowledgements}

I thank Dr Chi-Feng Lee (TARI), Dr Masahiro Ohara (SEHU), Dr Junsuke Yamasako (University of Tokyo), Dr Kiyoshi Ando (EUMJ), Dr Matthias Hartmann (NME), Mr Masato Mori and Mr Kenji Kitayama (Osaka) for supplying the material used in this paper, Mr Hideki Kinjo (Orion Breweries, Ltd.) for permission to use the name of the company and Mr. Dennis Murphy (The United Graduate School of Agricultural Sciences, Ehime University) for his critical reading of the draft. This study is supported in part by KAKENHI (24405028; principal investigator: S. Okajima). 


\section{References}

Champion G.C. 1923. Some Indian Coleoptera (12). Entomologist's Monthly Magazine 59: 219-224.

Hernando C. \& Ribera I. 2000. Taxonomic revision of the Afrotropical genus Cyclolimnichus Delève (Coleoptera: Limnichidae). African Entomology 8: 211-216.

Hernando C. \& Ribera I. 2001. Tricholimnichus gen. n. and three new species from Borneo (Coleoptera: Limnichidae). Koleopterologische Rundschau 71: 153-161.

Hernando C. \& Ribera I. 2005a. Pseudothryptus, a new genus of Limnichidae (Coleoptera) for Caccothryptus multiseriatus. Entomological Problems 35 (2): 131-135.

Hernando C. \& Ribera I. 2005b. 18.5. Limnichidae Erichson, 1846. In: Beutel R.G. \& Leschen R.A.B. (eds) Handbook of Zoology, Volume IV (Part 38), Coleoptera, Beetles, Volume 1: Morphology and Systematics (Archostemata, Adephaga, Myxophaga, Polyphaga partim). Berlin, Walter de Gruyter.

Hernando C. \& Ribera I. 2006. Resachus Delève: new faunistic records, and description of a new species from Madagascar. Koleopterologische Rundschau 76: 367-371.

Hernando C. \& Ribera I. 2014. Taxonomic revision of the genus Caccothryptus Sharp (Coleoptera: Limnichidae). Koleopterologische Rundschau 84: 281-304.

Spangler P.J., Staines C.L., Spangler P.M. \& Staines S.L. 2001. A checklist of the Limnichidae and the Lutrochidae (Coleoptera) of the world. Insecta Mundi 15 (3): 151-165.

Manuscript received: 3 July 2015

Manuscript accepted: 13 August 2015

Published on: 26 October 2015

Topic editor: Koen Martens

Desk editor: Kristiaan Hoedemakers

Printed versions of all papers are also deposited in the libraries of the institutes that are members of the EJT consortium: Muséum National d'Histoire Naturelle, Paris, France; Botanic Garden Meise, Belgium; Royal Museum for Central Africa, Tervuren, Belgium; Natural History Museum, London, United Kingdom; Royal Belgian Institute of Natural Sciences, Brussels, Belgium; Natural History Museum of Denmark, Copenhagen, Denmark. 


\section{Appendix}

Updated list of Caccothryptus species of the world.

\section{The compactus species group}

Caccothryptus compactus Sharp, 1902 [Borneo]

urn:Isid:zoobank.org:act:23A5960B-838E-4704-B212-776A2A46F9AA

Caccothryptus maculosus (Pic, 1923) [Cambodia, China (Hainan), India (Andaman Isls), Indonesia (Bali, Java, Nias Isls), Laos, Myanmar, Thailand, Vietnam]

urn:1sid:zoobank.org:act:30E26245-0801-4CA0-ABBA-1ACBEC3D36B6

Caccothryptus schuhi Hernando \& Ribera, 2014 [Indonesia (Java)]

urn:Isid:zoobank.org:act:E0F9014E-F7C0-431A-8CA7-B39B52DD22E4

Caccothryptus sulawesianus Hernando \& Ribera, 2014 [Indonesia (Sulawesi)]

urn:1sid:zoobank.org:act:5CDCD8B8-F635-45AD-AF77-BA211018E906

\section{The rouyeri species group}

Caccothryptus rouyeri (Pic, 1922) [Indonesia (Sumatra)]

urn:1sid:zoobank.org:act:B91A9867-DE1D-4F52-A259-29227FF3F6EC

\section{The testudo species group}

Caccothryptus chayuensis sp. nov. [Tibet]

urn:1sid:zoobank.org:act:43136CDF-3F5C-4AD4-A7DC-3F57299013EC

Caccothryptus auratus Hernando \& Ribera, 2014 [Thailand]

urn:1sid:zoobank.org:act:7628FE0D-7166-4856-BE9F-3D87B4997AC6

Caccothryptus fujianensis Hernando \& Ribera, 2014 [China (Fujian)]

urn:1sid:zoobank.org:act:2F1D4CE4-1FBC-4402-B30C-A850319297E6

Caccothryptus jendeki Hernando \& Ribera, 2014 [India]

urn:Isid:zoobank.org:act:0FC41492-8F47-4A7A-B653-F9BC4D934411

Caccothryptus malickyi Hernando \& Ribera, 2014 [Vietnam]

urn:1sid:zoobank.org:act:97038F29-11C2-4B1C-B8FB-7BEA76CCF1B5

Caccothryptus nepalensis Hernando \& Ribera, 2014 [Nepal]

urn:1sid:zoobank.org:act:7282A34B-B2D6-4AC6-AC3B-01EEFDCEE046

Caccothryptus orion sp. nov. [Japan (Okinawa)]

urn:Isid:zoobank.org:act:42B7D5F0-7DC5-4BD5-8CCF-5A08F9222A54

Caccothryptus punctatus (Pic, 1923) [Vietnam]

urn:1sid:zoobank.org:act:DB30A2CD-2FA0-44B8-A483-73FA19998B1C

Caccothryptus ripicola Champion, 1923 [India, Nepal]

urn:Isid:zoobank.org:act:66B64DF7-DF24-40EF-A9E3-7FB4D6C570A7

Caccothryptus sinensis Hernando \& Ribera, 2014 [China (Fujian)]

urn:1sid:zoobank.org:act:80F56A12-8B39-4BB0-862E-EBF0B9045233 
Caccothryptus taiwanus sp. nov. [Taiwan] urn:Isid:zoobank.org:act:578B475A-54DA-4600-9FFF-F5E7045F814E

Caccothryptus testudo Champion, 1923 [India, Nepal, Thailand] urn:Isid:zoobank.org:act:297F420C-723C-4B7D-822C-E0FBAB7ACB23

Caccothryptus tibetanus sp. nov. [Tibet] urn:lsid:zoobank.org:act:3CE4012A-96D7-4568-9DB7-F2175F0B1278

\section{The jaechi species group}

Caccothryptus jaechi Hernando \& Ribera, 2014 [Indonesia (Sulawesi)] urn:1sid:zoobank.org:act:640F4532-CAEE-49DB-8B0D-3F4A792F77B0

Caccothryptus nanus Hernando \& Ribera, 2014 [Philippines (Luzon)] urn:1sid:zoobank.org:act:51E4301C-BD4A-4DD2-BCC4-ACDFBD9617F1

Caccothryptus ticaoensis Hernando \& Ribera, 2014 [Philippines (Ticao)] urn:1sid:zoobank.org:act:8274ACEB-FEA7-45BF-8BBD-21EF3867D26D

Caccothryptus wooldridgei Hernando \& Ribera, 2014 [Indonesia (Sulawesi)] urn:1sid:zoobank.org:act:E87BD693-124E-4368-87EC-68867D88A62A

\section{The zetteli species-group}

Caccothryptus luzonensis Hernando \& Ribera, 2014 [Philippines (Luzon, Marinduque, Mindanao)] urn:Isid:zoobank.org:act:C487E48B-4CBD-4411-90F7-5EA7E4419150

Caccothryptus zetteli Hernando \& Ribera, 2014 [Philippines (Luzon)] urn:Isid:zoobank.org:act:7BDBC60A-D774-4782-8855-1ACEB762A1D7 